\title{
Regulatory T cells-an important target for cancer immunotherapy
}

Jae II Shin and Sang-Jun $\mathrm{Ha}$

A recent article by Drake et al. (Breathing new life into immunotherapy: review of melanoma, lung and kidney cancer. Nat. Rev. Clin. Oncol. 11, 24-37 [2014]) ${ }^{1}$ reviewed how monoclonal antibodies against the immune checkpoint molecules cytotoxic T-lymphocyte antigen-4 (CTLA-4), programmed death-1 (PD-1) and PD-ligand 1 (PD-L1) are effective in mediating tumour shrinkage in several cancer types. Drake et al. ${ }^{1}$ also highlighted ongoing phase III clinical trials and discussed the increased antitumour activity of the combinations of these antibodies compared with blocking either checkpoint alone. However, we would like to note that regulatory $\mathrm{T}$ cells $\left(\mathrm{T}_{\mathrm{REG}}\right)$ might represent another important immunological checkpoint to target in cancer immunotherapy. ${ }^{2,3}$ In fact, $\mathrm{T}_{\mathrm{REG}}$ that express $\mathrm{CD} 4$, $\mathrm{CD} 25$ and the forkhead protein 3 (FoxP3) can inhibit the antitumour immune response, thereby limiting the power of cancer immunotherapies. ${ }^{2}$

Although Drake et al. ${ }^{1}$ did not discuss the effects of CTLA- 4 and PD- 1 or PD-L1 blockade on $\mathrm{CD}^{+}{ }^{+} \mathrm{CD} 25^{+} \mathrm{FoxP}^{+} \mathrm{T}_{\mathrm{REG}}$, this needs to be considered. PD-1 blockade negatively regulates intracellular FoxP3 expression in $\mathrm{T}_{\mathrm{REG}}{ }^{4,5}$ Specifically, Wang et al. ${ }^{4}$ showed that PD-1 blockade leads to the downregulation of intracellular FoxP3 expression in $\mathrm{T}_{\mathrm{REG}}$ of patients with melanoma, suggesting that $\mathrm{PD}-1$ is implicated in the regulation of $\mathrm{T}_{\mathrm{REG}}$ function. Furthermore, Sharma et al. ${ }^{5}$ demonstrated that the ability of $\mathrm{T}_{\mathrm{REG}}$ to suppress target $\mathrm{T}$-cell proliferation is abrogated by PD- 1 and PD-L1 antibodies. The effect of CTLA- 4 blockade on $\mathrm{T}_{\mathrm{REG}}$ is less clear. ${ }^{6,7}$ CTLA-4 is constitutively expressed on CD4+ $\mathrm{T}_{\mathrm{REG}}{ }^{8,9}$ and Wing et al. ${ }^{8}$ reported that $\mathrm{T}_{\mathrm{REG}}$-specific CTLA-4 deficiency impaired in vivo and in vitro suppressive function of $\mathrm{T}_{\mathrm{REG}}$, and also produced potent tumour immunity. Regarding the effect of CTLA-4 blockade on $\mathrm{T}_{\text {REG, }}$ Kavanagh et al. ${ }^{6}$ showed that treatment with CTLA-4 antibodies in patients with metastatic prostate cancer induces an increase in the number of activated effector $\mathrm{CD} 4^{+} \mathrm{T}$ cells and $\mathrm{CD} 4^{+} \mathrm{CD} 25^{+} \mathrm{FoxP} 3^{+}$ $\mathrm{T}_{\mathrm{REG}}$, suggesting that CTLA- 4 antibodies enhance antitumour immunity by the activation of effector $\mathrm{T}$ cells rather than by depleting $\mathrm{CD} 4^{+} \mathrm{CD} 25^{+} \mathrm{FoxP}^{+} \mathrm{T}_{\mathrm{REG}}$ in vivo. ${ }^{6}$ Conversely, Simpson et al. ${ }^{7}$ reported that treatment with an anti-CTLA-4 antibody induces a selective depletion of $\mathrm{T}_{\mathrm{REG}}$ within the tumour lesions in a mouse model of melanoma.

On the basis of the different effects that immune checkpoint blockade can have on $\mathrm{T}_{\mathrm{REG}}$ function, it is possible that circumventing the activity of $\mathrm{T}_{\mathrm{REG}}$ might represent an important step to overcome some of the obstacles that, so far, have prevented the complete exploitation of the immunotherapy potential for the successful treatments of many cancers, including melanoma. ${ }^{3}$ A substantial number of $\mathrm{T}_{\mathrm{REG}}$, in tumour tissues and peripheral blood specifically express $\mathrm{C}-\mathrm{C}$ chemokine receptor 4 (CCR4), therefore, treatment with antiCCR4 monoclonal antibody can evoke and augment antitumour immunity in patients with melanoma by selectively depleting or inhibiting $\mathrm{T}_{\mathrm{REG}}$ from the tumour tissue. ${ }^{10,11}$ The combinations of CTLA- 4 and PD- 1 or PD-L1 blockade showed an increased antitumour immunity compared with using each antibody alone; ${ }^{1}$ however, an alternative combination strategy could include both immune checkpoint blocking antibodies and $\mathrm{T}_{\mathrm{REG}}$-depleting molecules. For example, depletion of $\mathrm{T}_{\mathrm{REG}}$ by intraperitoneal administration of interleukin-2 diphtheria toxin followed by sequential PD-1 or PD-L1 blockade showed superior efficacy for eradication of acute myeloid leukaemia in a mouse model than did PD-1 or PD-L1 blockade alone. ${ }^{12}$ Furthermore, Goding et al. ${ }^{13}$ demonstrated that either blockade of the PD-1 pathway with anti-PD-L1 antibodies or depletion of tumour-specific $\mathrm{T}_{\mathrm{REG}}$ alone did not prevent tumour recurrence in a mouse model of melanoma. However, the combination of PD-L1 blockade and intumour $\mathrm{T}_{\mathrm{REG}}$ depletion via administration of interleukin-2 diphtheria toxin effectively mediated melanoma regression. ${ }^{13}$ These results indicate that primary and relapsing cancer might have different characteristics and the use of combined immunotherapy approaches that specifically target $\mathrm{T}_{\mathrm{REG}}$ cells, could be required for highly resistant recurrent disease.

Overall, we believe that the effect of immune checkpoint blockade (via CTLA-4, PD-1 or PD-L1) on $\mathrm{T}_{\mathrm{REG}}$ should be considered when evaluating the efficacy of cancer-immunotherapy and that the available data clearly warrant clinical studies of combined immune checkpoint blockade and a $\mathrm{T}_{\mathrm{REG}}$-targeting strategy in primary and relapsed cancers.

Department of Paediatrics, Yonsei University College of Medicine, 50 Yonsei-ro,

Seodaemun-gu, C.P.O. Box 8044 ,

Seoul 120-752, Republic of Korea (J.I.S.).

Department of Biochemistry, College of Life Science and Biotechnology, Yonsei University, Seoul 120-749, Republic of Korea (S.-J.H.). Correspondence to: J.I.S.

shinji@yuhs.ac

\section{Competing interests}

The authors declare no competing interests.

1. Drake, C. G., Lipson, E. J. \& Brahmer, J. R. Breathing new life into immunotherapy: review of melanoma, lung and kidney cancer. Nat. Rev. Clin. Oncol. 11, 24-37 (2014).

2. Waldmann, T. A. Effective cancer therapy through immunomodulation. Annu. Rev. Med. 57, 65-81 (2006).

3. Pandolfi, F. et al. Strategies to overcome obstacles to successful immunotherapy of melanoma. Int. J. Immunopathol. Pharmacol. 21, 493-500 (2008).

4. Wang, W. et al. PD1 blockade reverses the suppression of melanoma antigen-specific CTL by $\mathrm{CD} 4+\mathrm{CD} 25(\mathrm{Hi})$ regulatory $\mathrm{T}$ cells. Int. Immunol. 21, 1065-1077 (2009).

5. Sharma, M. D. et al. Plasmacytoid dendritic cells from mouse tumor-draining lymph nodes directly activate mature Tregs via indoleamine 2,3-dioxygenase. J. Clin. Invest. 117, 2570-2582 (2007).

6. Kavanagh, B. et al. CTLA4 blockade expands FoxP3+ regulatory and activated effector CD4+ T cells in a dose-dependent fashion. Blood 112, 1175-1183 (2008). 


\section{CORRESPONDENCE}

7. Simpson, T. R. et al. Fc-dependent depletion of tumor-infiltrating regulatory $T$ cells co-defines the efficacy of anti-CTLA-4 therapy against melanoma. J. Exp. Med. 210, 1695-1710 (2013).

8. Wing, K. et al. CTLA-4 control over Foxp3+ regulatory $T$ cell function. Science 322 271-275 (2008)

9. Sakaguchi, S. et al. FOXP3+ regulatory T cells in the human immune system. Nat. Rev. Immunol. 10, 490-500 (2010).
10. Sugiyama, D. et al. Anti-CCR4 mAb selectively depletes effector-type FoxP3+CD4+ regulatory T cells, evoking antitumour immune responses in humans. Proc. Natl Acad. Sci. USA 110, 17945-17950 (2013).

11. Pere, $\mathrm{H}$. et al. A CCR4 antagonist combined with vaccines induces antigen-specific CD8+ T cells and tumour immunity against self antigens. Blood 118, 4853-4862 (2011).
12. Zhou, Q. et al. Program death-1 signalling and regulatory $T$ cells collaborate to resist the function of adoptively transferred cytotoxic T Iymphocytes in advanced acute myeloid leukemia. Blood 116, 2484-2493 (2010).

13. Goding, S. R. et al. Restoring immune function of tumour-specific CD4+ T cells during recurrence of melanoma. J. Immunol. 190, 4899-4909 (2013) 